\title{
Radiative and dynamic stability of a dilute plasma
}

\author{
Steven A. Balbus ${ }^{1,2}$, Christopher S. Reynolds ${ }^{3,4}$
}

\begin{abstract}
We analyze the linear stability of a dilute, hot plasma, taking into account the effects of stratification and anisotropic thermal conduction. The work is motivated by attempts to understand the dynamics of the intracluster medium in galaxy clusters. We show that magnetic field configurations that nominally stabilize either the heat-flux driven buoyancy instability (associated with a positive thermal gradient) or the magnetothermal instability (negative thermal gradient) can lead to previously unrecognized $g$-mode overstabilities. The driving source of the overstability is either radiative cooling (positive temperature gradient) or the heat flux itself (negative temperature gradient). While the implications of these overstabilities have yet to be explored, we speculate that the cold fronts observed in many relaxed galaxy clusters may be related to their non-linear evolution.
\end{abstract}

Subject headings: instabilities — plasmas — magnetohydrodynamics (MHD) convection — galaxies: clusters: intracluster medium

\section{Introduction}

The dynamical stability properties of a dilute plasma change dramatically when even a weak magnetic field with no " $J \times B$ " force is present. This is a consequence of anisotropic transport: if the mean free path exceeds the relevant gyroradius, heat and (angular) momentum are transported only along magnetic lines of force (Balbus 2000, 2004). In particular, in a stratified atmosphere of dilute plasma, the Schwarzschild stability criterion (i.e. specific entropy must increase upwards in a gravitational potential) no longer governs convective stability (Balbus 2001). Instead, the presence of any temperature gradient is potentially

\footnotetext{
${ }^{1}$ Laboratoire de Radioastronomie, École Normale Supérieure, 24 rue Lhomond, 75231 Paris CEDEX 05, France steven.balbus@lra.ens.fr

${ }^{2}$ Adjunct Professor, Department of Astronomy, University of Virginia, Charlottesville VA 22903

${ }^{3}$ Department of Astronomy, University of Maryland, College Park, MD20742

${ }^{4}$ Joint Space Science Institute (JSI), University of Maryland, College Park, MD20742
} 
convectively unstable, depending on the relative alignment of the magnetic field and thermal gradient (Quataert 2008, hereafter Q08). The only configurations that are nominally stable are i) an upwardly decreasing temperature profile whose gradient is precisely aligned with the magnetic field, and ii) a upwardly increasing temperature profile whose gradient is precisely orthogonal to the magnetic field. Any other configuration is unstable. In this Letter, we show that even these "stable" configurations are in fact subject to overstability. Interestingly, the non-linear evolution of the thermal gradient instabilities tends to drive the system towards these overstable states. In essence, a dilute stratified plasma that is hot at its core wants to let the heat escape; a cold-core system wants to self-insulate itself. The hot core instability is known as the magnetothermal instability (MTI). The cold core instability is known as the heat flux buoyancy instability (HBI), because the free energy source is the thermal flux itself.

Both the MTI and HBI may be of considerable importance for understanding the behavior of the intracluster medium (ICM) in clusters of galaxies. The outer regions of ICM atmospheres have a negative temperature gradient and MTI-driven turbulence may be important for energy and metal transport (Parrish, Stone \& Lemaster 2008). Furthermore, in clusters with cooling cores, radiative losses lead to the formation of a positive temperature gradient in the central regions of the ICM: a cool ICM core is embedded in a hotter ICM atmosphere (e.g., review by Peterson \& Fabian 2006). A longstanding problem has been to understand the thermal stability of the cool inner regions of ICM atmospheres. In particular, why do radiative losses not cause run away cooling in these regions? The standard paradigm is that regulated energy injection into the ICM by a central active galactic nucleus (AGN) is a stabilizing influence. However, the finding of Q08 the positive temperature gradient within the ICM of cooling core clusters could be convectively unstable suggests that the destabilzing role played by thermal conduction merits closer scrutiny in this context.

A major difficulty with invoking thermal conduction to stave off radiative core collapse of the ICM (e.g., Bertschinger \& Meiksen 1986, Zakamska \& Narayan 2003) has been the fine tuning required (Conroy \& Ostriker 2008). A little too much thermal conduction evaporates the cool core; too little allows the collapse to proceed essentially unimpeded. Balbus \& Reynolds (2008) hypothesized that the combination of thermal and radiative losses may be self-regulating. In this view, suppression of the thermal conduction is not complete. Instead, through field line tangling and a possible reverse HBI convective thermal flux, the radiative core is able to draw in the requiste heat flux for marginal stability. Global MHD models of cluster cores have recently tested these ideas (Bogdanovic et al. 2009; Parrish, Quataert \& Sharma, 2009, 2010; Ruszkowski \& Oh 2010). These simulations show that in idealized static atmospheres, while there is significant heat transport into the cooling core, after several cooling times the non-linear development of the HBI wraps the magnetic field onto shells, 
insulating the core from further conduction and fostering thermal collapse. Models with sufficiently strong forced turbulence (perhaps the effects of an AGN, or stirring by galaxy motions and/or sub-cluster mergers) can prevent the field-line wrapping and stabilize the cluster. However, such models predict temperature decrements in the core of at most a factor of two; real cooling core clusters often have much steeper temperature gradients. Moreover, the forced turbulence is assumed to be volume-filling, the justification for which is unclear.

In this Letter, we return to the fundamental question of the linear stability of an atmosphere of dilute weakly magnetized plasma in the presence of a background temperature gradient. We generalize the treatment of Q08 to include optically-thin radiative losses. Our principal result is the finding of new overstabilities of dynamical waves. More precisely, we find that nominally stable configurations resulting from the non-linear evolution of the HBI (i.e., temperature increasing upwards and magnetic field essentially horizontal) generate overstable $g$-modes via radiative losses. Nominally stable configurations resulting from the non-linear evolution of the MTI (i.e., temperature decreasing upwards and magnetic field essentially vertical) generate overstable $g$-modes via anisotropic thermal conduction. In addition to furnishing a more complete formal picture of the stability properties of dilute plasma

atmospheres, these findings may have significant implications for the physical behavior of the ICM, and should guide future simulations.

In the next section, we present the calculation in detail, and in the final section of this Letter, we conclude with a brief discussion of the implications of our findings.

\section{Analysis}

We use the standard equations of MHD, with the entropy equation agumented with anisotropic thermal conduction along magnetic field lines (Braginskii 1965) and radiative losses (e.g. Field 1965). The mass, momentum, induction, and entropy equations are respectively

$$
\begin{gathered}
\frac{\partial \rho}{\partial t}+\nabla \cdot(\rho \boldsymbol{v})=0, \\
\rho \frac{D \boldsymbol{v}}{D t}=\frac{(\boldsymbol{\nabla} \times \boldsymbol{B}) \times \boldsymbol{B}}{4 \pi}-\boldsymbol{\nabla}+\rho \boldsymbol{g}, \\
\frac{\partial \boldsymbol{B}}{\partial t}=\boldsymbol{\nabla} \times(\boldsymbol{v} \times \boldsymbol{B}), \\
\frac{D \ln P \rho^{-\gamma}}{D t}=-\frac{\gamma-1}{P}[\boldsymbol{\nabla} \cdot \boldsymbol{Q}+\rho \mathcal{L}],
\end{gathered}
$$

where $\rho$ is the mass density, $\boldsymbol{v}$ is the fluid velocity, $\boldsymbol{B}$ is the magnetic field, $\boldsymbol{g}$ is the gravitational acceleration, $P$ is the gas pressure, $\gamma$ is the adiabatic index (5/3 for monotonic gas), 
$\boldsymbol{Q}$ is the heat flux, and $\mathcal{L}$ is the radiative energy loss per unit mass of fluid, whose form we will leave unspecified. For thermal bremsstrahlung, a reasonable approximation is

$$
\rho \mathcal{L} \simeq 2 \times 10^{-27} n_{e}^{2} T^{1 / 2} \mathrm{ergs} \mathrm{cm}^{-3} \mathrm{~s}^{-1},
$$

where $n_{e}$ is the electron number density. $D / D t$ is the Lagrangian derivative, $\partial / \partial t+\boldsymbol{v} \cdot \boldsymbol{\nabla}$.

To define the heat flux $\boldsymbol{Q}$, let $\boldsymbol{b}$ be a unit vector in the direction of the magnetic field. Then (Balbus 2001):

$$
\boldsymbol{Q}=-\chi \boldsymbol{b}(\boldsymbol{b} \cdot \boldsymbol{\nabla}) T,
$$

where $T$ is the kinetic gas temperature and $\chi$ is the thermal conductivity (Spitzer 1962):

$$
\chi \simeq 6 \times 10^{-7} T^{5 / 2} \mathrm{ergs} \mathrm{cm}^{-1} \mathrm{~s}^{-1} \mathrm{~K}^{-1} .
$$

Finally, we follow Q08 and use the notation

$$
\kappa \equiv \chi T / P
$$

for the thermal diffusion coefficient.

\subsection{Equilibrium Background}

We consider a gas stratified in the vertical $z$ direction, with temperature profile $T(z)$. The gas is not self-gravitating, so that $\boldsymbol{g}$ is a specified function of position. We assume a highly sub-thermal magnetic field. Thus, in equilibrium, the gas is in hydrostatic balance,

$$
\frac{d P}{d z}=-\rho g
$$

The magnetic field is uniform with $x$ and $z$ components $B_{x}$ and $B_{z}$, (in this way defining the $x$ axis), and unit vectors $b_{x}=B_{x} / B, b_{z}=B_{z} / B$. In equilibrium, there is a thermal balance between conductive heating and radiative losses,

$$
-\nabla \cdot \boldsymbol{Q} \equiv \frac{d^{2}\left(b_{z}^{2} \chi\right) T}{d z^{2}}=\rho \mathcal{L} .
$$

\subsection{Local WKB Perturbations}

As in Q08, we consider plane wave disturbances of the form $\exp (\sigma t+i \boldsymbol{k} \cdot \boldsymbol{r})$ where the wavenumber $\boldsymbol{k}$ has Cartesian components $\left(k_{x}, k_{y}, k_{z}\right)$, and $\boldsymbol{r}$ is the position vector. We differ 
in notation from Q08 by using $\sigma$, a formal growth rate, rather than $\omega$, an angular frequency. This ensures that all coefficients in the final dispersion relation are real. We work in the WKB $(k r \gg 1)$ and Boussinesq limits (Q08).

We next consider the linearized equations when perturbations $\delta \rho, \delta \boldsymbol{v}$, etc., are applied to the equilibrium state. The heart of the problem is the entropy equation, so let us begin here. The linearized form of equation (4) is

$$
-\gamma \sigma \frac{\delta \rho}{\rho}+\delta v_{z} \frac{d \ln P \rho^{-\gamma}}{d z}=(\gamma-1)\left[-\frac{\boldsymbol{\nabla} \cdot \delta \boldsymbol{Q}}{P}-\Theta_{T \mid P} \delta T\right],
$$

where

$$
\Theta \equiv \rho \mathcal{L} / P
$$

and

$$
\Theta_{T \mid P} \equiv\left[\frac{\partial \Theta}{\partial T}\right]_{P},
$$

that is, the derivative of $\Theta$ with respect to $T$ with $P$ held constant. We have used the Boussinesq approximation in ignoring all terms proportional to $\delta P$ in equation (11). In the process, we have implicitly regarded $\Theta$ as a function of $T$ and $P$ (rather than the more customary but less convenient $T$ and $\rho$ dependence). The remaining linearized equations,

$$
\begin{gathered}
\boldsymbol{k} \cdot \boldsymbol{v}=0 \\
\sigma \delta \boldsymbol{v}=\frac{\delta \rho}{\rho^{2}} \frac{d P}{d z}-i \boldsymbol{k}\left(\frac{\delta P}{\rho}+\frac{\boldsymbol{B} \cdot \delta \boldsymbol{B}}{4 \pi \rho}\right)+\frac{i(\boldsymbol{k} \cdot \boldsymbol{B}) \delta \boldsymbol{B}}{4 \pi \rho} \\
\sigma \delta \boldsymbol{B}=i(\boldsymbol{k} \cdot \boldsymbol{B}) \delta \boldsymbol{v}
\end{gathered}
$$

are, apart from notational convention, identical to Q08. The entire system of equations differs from Q08 only by the $\Theta$ term. The resulting dispersion relation is

$$
\begin{array}{r}
\left(\sigma+\frac{\gamma-1}{\gamma} T \Theta_{T \mid P}+\mathcal{C}\right)\left(\sigma^{2}+\left(\boldsymbol{k} \cdot \boldsymbol{v}_{\boldsymbol{A}}\right)^{2}\right) \\
+\frac{\sigma k_{\perp}^{2} N^{2}}{k^{2}}+\mathcal{C K} \frac{g}{k^{2}} \frac{d \ln T}{d z}=0
\end{array}
$$

where

$$
\begin{gathered}
N^{2}=-\frac{1}{\rho \gamma} \frac{d P}{d z} \frac{d \ln P \rho^{-\gamma}}{d z}=g \frac{d \ln P^{(1-\gamma) / \gamma} T}{d z} \\
\mathcal{C}=\left(\frac{\gamma-1}{\gamma}\right) \kappa(\boldsymbol{k} \cdot \boldsymbol{b})^{2} \\
\mathcal{K}=\left(1-2 b_{z}^{2}\right) k_{\perp}^{2}+2 b_{x} b_{z} k_{x} k_{z} .
\end{gathered}
$$


This corresponds to equation (13) of Q08, except, as noted, for the single appearance of the radiative $\Theta_{T \mid P}$ term. (A less general version of this result was also presented in Balbus \& Reynolds [2008].) The dispersion characterizes the linear response of a magnetized, thermally conducting radiative dilute plasma to incompressible disturbances.

\subsection{Stability}

\subsubsection{Recovery of the Conductive Field criterion}

Expanding the dispersion relation (17) leads to

$$
\sigma^{3}+a_{1} \sigma^{2}+a_{2} \sigma+a_{3}=0
$$

where

$$
\begin{gathered}
a_{1}=\left(\frac{\gamma-1}{\gamma}\right) T \Theta_{T \mid P}+\mathcal{C}, \\
a_{2}=\frac{k_{\perp}^{2}}{k^{2}} N^{2}+\left(\boldsymbol{k} \cdot \boldsymbol{v}_{\boldsymbol{A}}\right)^{2}, \\
a_{3}=\mathcal{C} \mathcal{K} \frac{g}{k^{2}} \frac{d \ln T}{d z}+\left(\boldsymbol{k} \cdot \boldsymbol{v}_{\boldsymbol{A}}\right)^{2} a_{1} .
\end{gathered}
$$

There are stable solutions to this dispersion relation if and only if the following three criteria are met:

$$
a_{1}>0, \quad a_{3}>0, \quad a_{1} a_{2}>a_{3} .
$$

This follows from a Routh-Hurwitz analysis, but can be seen more easily by inspection: the first two are in fact elementary, while the third follows from self-consistently demanding purely imaginary solutions to the cubic equation and then investigating their behavior for infinitesimal real parts.

The physical interpretation of $a_{1}>0$, or

$$
T \Theta_{T \mid P}+(\boldsymbol{k} \cdot \boldsymbol{b})^{2} \kappa>0,
$$

is the magnetized conduction variation of the classical thermal instability criterion (Field 1965). Only the component of $k$ along the field lines enters into the conduction term.

\subsubsection{Recovery of the HBI and MTI}

We next consider the physical interpretation of $a_{3}>0$, or

$$
\mathcal{C K} \frac{g}{k^{2}} \frac{d \ln T}{d z}+\left(\boldsymbol{k} \cdot \boldsymbol{v}_{\boldsymbol{A}}\right)^{2} a_{1}>0 .
$$


In essence, this is the HBI/MTI criterion of Q08, but further (de)stabilized when the flow is (un)stable by the isobaric Field criterion. This is a true instability if $a_{3}$ is negative, with $\sigma=-a_{3} / a_{2}$ in the limit of large $a_{2}>0$.

Equation (27) shows that thermal instability and the HBI/MTI are intimately linked. To be definite, consider the behavior of the HBI. The discussion of Q08 explains how the distortion of field lines leads to conductive cooling of a downwardly displaced fluid element (and vice-versa for an upwardly displaced element). It is this cooling that causes the convection associated with the HBI. With radiative losses present, the cooling is enhanced on a downward displacement, and relative heating is present on an upward displacement. In fact, we may imagine now slowly turning on the magnetic field from dynamically weak to strongly dominant. Then, the exponentially growing instability transforms from the Q08 HBI to the classical (nonoscillatory) thermal instability. The role of the magnetic field in mediating this transition is crucial.

\subsubsection{Destabilization of wave modes by a positive thermal gradient}

We now return to the third criterion, $a_{1} a_{2}>a_{3}$. With $a_{3}>0$, this criterion is a more stringent stability criterion than the first $\left(a_{1}>0\right)$, and hence replaces it.

When $a_{2}$ is large and positive [e.g. either $N^{2}$ or $\left(\boldsymbol{k} \cdot \boldsymbol{v}_{\boldsymbol{A}}\right)^{2}$ is dominant], the unstable roots depending on $a_{1}$ will be approximately:

$$
\sigma= \pm i a_{2}^{1 / 2}+\left(a_{3}-a_{1} a_{2}\right) / 2 a_{2}
$$

On the other hand, at large wavenumbers, we may have $a_{1}$ and $a_{3}$ as the dominant terms. If $a_{3}$ and $a_{1}$ are both positive (or both negative), then the wavelike solutions will be

$$
\sigma= \pm i\left(a_{3} / a_{1}\right)^{1 / 2}+\left(a_{3}-a_{1} a_{2}\right) / 2 a_{1}^{2}
$$

In either case above, the combination $a_{3}-a_{1} a_{2}$ determines the stability of the mode.

After a cancellation of the magnetic tension terms, the condition $a_{1} a_{2}-a_{3}>0$ becomes

$$
a_{1} \frac{k_{\perp}^{2}}{k^{2}} N^{2}-\mathcal{C K} \frac{g}{k^{2}} \frac{d \ln T}{d z}>0 .
$$

Consider the limit $b_{z} \ll 1$, which is HBI stable $\left(a_{3}>0\right)$ for all but nearly axial wavenumbers, whose growth times then become very long. (We cannot take $b_{z}=0$ exactly, since that would preclude a static radiative equilibrium state. For $b_{z}$ finite, equation (10) shows that the equilibrium $d T / d z$ scales as $b_{z}^{-1}$.) Then, $\mathcal{K}=k_{\perp}^{2}$, and our inequality reduces to

$$
\frac{\gamma-1}{\gamma} T \Theta_{T \mid P} N^{2}+\mathcal{C}\left(N^{2}-g \frac{d \ln T}{d z}\right)>0 .
$$


But

$$
N^{2}-g \frac{d \ln T}{d z}=\frac{\gamma-1}{\gamma} \frac{1}{P \rho}\left(\frac{d P}{d z}\right)^{2},
$$

and assuming that $N^{2}>0$, the inequality may be yet further reduced:

$$
T \Theta_{T \mid P}+\frac{\mathcal{C}}{\rho P N^{2}}\left(\frac{d P}{d z}\right)^{2}>0 .
$$

Finally, substituting for $\mathcal{C}$ and $N^{2}$ and simplifying, our condition becomes

$$
T \Theta_{T \mid P}+\kappa(\boldsymbol{k} \cdot \boldsymbol{b})^{2} \mathcal{R}>0,
$$

where $\mathcal{R}$ is the reduction factor

$$
\mathcal{R}=\left(1+\left|\frac{\gamma}{\gamma-1} \frac{d \ln T}{d \ln P}\right|\right)^{-1},
$$

which bears direct comparison with equation (26). Here, thermal conduction once again stabilizes radiative losses, but the HBI terms, when they are in a stable configuration relative to the convective processes discussed by Q08, actively destabilize wave-like modes by reducing the supression of thermal conduction. In regions of sharp temperature gradients, the effective reduction factor for conductive stabilization can be large. Indeed, in the chosen limit $b_{z} \ll 1$, we have $\mathcal{R} \sim O\left(b_{z}\right)$. Note that wavenumbers with vanishing $\boldsymbol{k} \cdot \boldsymbol{b}$ are unaffected by conduction, and have an effective reduction factor of zero. In our example, these are horizontal fluid displacements along the magnetic field lines.

\subsubsection{Destabilization of wave modes by a negative thermal gradient}

Consider next the case $b_{z}=1$, which would be HBI-unstable in the case of an increasing outward temperature profile. But let us now assume that the temperature decreases outwards. This configuration is HBI stable. With $b_{z}=1$, if we restricted ourselves only to the first two stability criteria, we would conclude that this configuration is also MTI-stable. In fact, if the third stability criterion is imposed, this configuration is subject to an interesting and powerful overstability, driven by anisotropic thermal conduction, as we now show.

With $b_{z}=1$ we have $\mathcal{K}=-k_{\perp}^{2}$ and our third criterion inequality (30) becomes

$$
\frac{\gamma-1}{\gamma} T \Theta_{T \mid P} N^{2}+\mathcal{C}\left(N^{2}+g \frac{d \ln T}{d z}\right)>0 .
$$


Once again, the thermal conduction is affected by a "reduction factor," though here the reduction factor $\mathcal{R}^{\prime}$ actively destabilizes rather than merely supresses dissipative destabilization. The above inequality may be written

$$
T \Theta_{T \mid P}+\kappa(\boldsymbol{k} \cdot \boldsymbol{b})^{2} \mathcal{R}^{\prime}>0
$$

where

$$
\mathcal{R}^{\prime}=1-\left[\frac{\gamma-1}{\gamma} \frac{d \ln P}{d \ln T}-1\right]^{-1}
$$

The term inside the square brackets must always be positive if $N^{2}>0$, but if

$$
1<\frac{\gamma-1}{\gamma} \frac{d \ln P}{d \ln T}<2
$$

then $\mathcal{R}^{\prime}<0$ and buoyant modes are overstable, even if there is no radiative loss term.

\subsubsection{Summary}

The MTI and HBI are evansecent instabilities present in dilute plasmas when anisotropic heat flux is included in the physics. The MTI is present when the thermal gradient decreases outward and the field lines are insulating in the equilibrium configuration. When the field lines open, the MTI is stabilized. The HBI is present when the thermal gradient increases outward and the field lines are open so that a heat flux is present in the equilibrium configuration. The action of the HBI is to close the field lines, which stabilizes the system.

We have found that the stable "end states" of these instabilities are subject to further overstabilities. In the case of the HBI, which is relevant for the coooling flow cores, a thermally unstable radiative loss function and closed fields lines together manifest as over stable buoyant oscillations. In the case of the MTI, a sufficiently steep (but classically convectively stable) outwardly decreasing thermal gradient produces overstable buoyant waves when the magnetic field lines are open and conducting heat.

The overstabilities nominally depend on radiative losses, but their effect should be thought of as dynamical: these are classical g-waves that in principle could be driven to finite amplitudes on thermal time scales (either radiative or conductive). Whether they are best thought of a local WKB waves, global modes, or both is not yet clear, and awaits numerical investigation. 


\section{Discussion and Conclusions}

The implications of the Q08 finding that generic cluster (or elliptical galaxy) cooling flows are convectively unstable have yet to be grasped. A more complete linear theory is clearly a starting point. Here, we have generalized the linear theory of such systems to include the effects of both anisotropic thermal conduction and optically-thin radiative losses.

To recap, strict stability requires three criteria to be satisfied. The first amounts to the classical Field criterion for thermal instability in the presence of anisotropic conduction,

$$
a_{1} \equiv T \Theta_{T \mid P}+\kappa(\boldsymbol{k} \cdot \boldsymbol{b})^{2}>0 \quad \text { (Stability) }
$$

The second criterion gives the MTI or HBI stability conditions depending upon the orientation of the magnetic field (via $\mathcal{K}$ ) and the temperature gradient,

$$
\mathcal{C K} \frac{g}{k^{2}} \frac{d \ln T}{d z}+\left(\boldsymbol{k} \cdot \boldsymbol{v}_{\boldsymbol{A}}\right)^{2} a_{1}>0 \quad \text { (Stability). }
$$

The third criterion has not, to our knowledge, been recognized previously. For the two limiting cases considered in this work, it takes the form

$$
\begin{array}{ll}
T \Theta_{T \mid P}+\kappa(\boldsymbol{k} \cdot \boldsymbol{b})^{2} \mathcal{R}>0 & \left(\text { Stability ; } b_{z} \approx 0 ; d T / d r>0\right), \\
T \Theta_{T \mid P}+\kappa(\boldsymbol{k} \cdot \boldsymbol{b})^{2} \mathcal{R}^{\prime}>0 & \left(\text { Stability ; } b_{z} \approx 1 ; d T / d r<0\right),
\end{array}
$$

where $0<\mathcal{R}<1$ and $-\infty<\mathcal{R}^{\prime}<1$. Even once the HBI (MTI) has been stabilized by the formation of horizontal (vertical) magnetic fields during their non-linear evolution, the third criterion can be violated in some range of wavenumbers leading to overstable $g$-modes.

The mechanism of the overstability differs depending upon the setting. The overstability that would naturally follow HBI-driven evolution $(d T / d z>0$ and essentially horizontal field) is driven by the presence of Field-unstable radiative cooling. On the other hand, the overstability that would naturally follow MTI-driven evolution $(d T / d z<0$ and essentially vertical field) is driven by the anisotropic heat flux, and is present even in the absence of radiative losses.

Figure 1 indicates schematically the set of instabilities and overstabilities discussed in this paper. At a formal level, it is remarkable that the conductive heat flux can drive the atmosphere away from stability irrespective of the sign of the temperature gradient: only very shallow negative temperature gradients [such that $d \ln P / d \ln T>2 \gamma /(\gamma-1)$ ] are completely stable, and even here the overstability can be reinstated if Field-unstable radiative losses are present. 


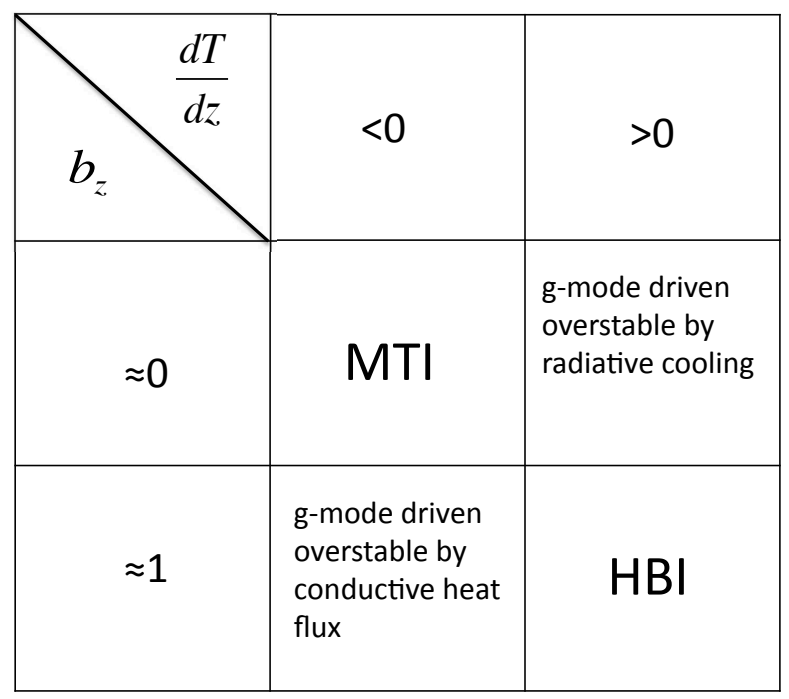

Fig. 1.- Schematic map of the instabilities and overstabilities discussed in this work.

The astrophysical implications of these overstabilities have yet to be determined, and we must await simulations in order to assess their global character as well as their non-linear behavioul 1 Here, we limit ourselves to some brief remarks and speculation. Probably the most important implication of these overstabilities for cooling core clusters is that, provided that radiative losses maintain a temperature gradient, the ICM may never be able to achieve a state that is stable to the HBI and the $g$-mode overstability. This statement holds true even if some unspecified heat source is fending off core collapse, provided that the net cooling function is still Field-unstable. The $g$-mode overstability grows on the cooling timescale and can be easily seeded by perturbations resulting from AGN activity, galaxy motions, sub-cluster mergers or indeed a preceeding phase of HBI-driven turbulence (Ruszkowski $\&$ Oh 2010). The $g$-mode overstability may still be relevant even when forced turbulence erases any obvious manifestations of the HBI. It is interesting to speculate that the "cold

\footnotetext{
${ }^{1}$ To date, published local studies of the HBI have excluded radiative cooling, and so are not be subject to this overstability. The overstabilities should, however, be present in published global studies of cooling cores (Bogdanovic et al. 2009; Parrish et al. 2009). But these simulated cores undergo a thermal runaway after several cooling times, and the overstabilities may be buried in the complex background dynamics, and very difficult to extract.
} 
fronts" seen in the ICM cores of many relaxed clusters (Markevitch et al. 2000; Ascasibar \& Markevitch 2006) may be related to the non-linear development of these radiatively-driven $g$-mode overstabilities.

At the purely formal level, understanding the development of thermal instability in a dilute plasma (Field 1965) has turned into a four decade struggle. And one speaks here of linear stability! It is a problem of some subtlety. With the advent of the HBI and our deepened understanding of the role of anisotropic conduction, we may at last have the true essence of the problem. There may, on the other hand, be more to come.

\section{Acknowledgements}

CSR thanks the Physics Department of the École Normale Supérieure de Paris for its hospitality and support of a one month visit in June 2010 during which some of this work was conducted. This work is supported by the NSF under grant AST 09-08212.

\section{REFERENCES}

Ascasibar, Y., \& Markevitch, M. et al., 2006, ApJ, 650, 102

Balbus, S.A. 2000, ApJ, 534, 420

Balbus, S.A. 2001, ApJ, 562, 909

Balbus, S.A. 2004, ApJ, 616, 857

Balbus S.A., \& Reynolds, C.S. 2008, ApJ, 681, L65

Betschinger, E., \& Meiksen, A. 1986, ApJ, 306, L1

Bogdanović, T., Reynolds, C.S., Balbus, S.A., Parrish, I.J., 2009, ApJ, 704, 211

Braginskii, S.I. 1965, Rev. Plasma Phys., 1, 205

Conroy, C., \& Ostriker, J.P., ApJ, 681, 151

Field, G.B. 1965, ApJ, 142, 531

Markevitch, M. et al., 2000, ApJ, 541, 542

Parrish, I.J., \& Quataert, E. 2008, ApJ, 677, L9 
Parrish, I.J., Quataert, E., Sharma, P., 2009, ApJ, 703, 96

Parrish, I.J., Quataert, E., Sharma, P., 2010, ApJ, 712, L194

Parrish, I.J., Stone, J.M. \& Lemaster, N.. 2008, ApJ, 688, 905

Peterson, J.R., \& Fabian, A.C., 2006, Physics Reports, 427, 1

Ruszkowski, M., Oh, S.P., 2010, ApJ, 713, 1332

Quataert, E. 2008, ApJ, 673, 758 (Q08)

Zakamska, N.L., \& Narayan, R. 2003, ApJ, 582, 162 